\title{
RULE BASED PSEUDO N-GRAM MODEL FOR TELUGU SCRIPT
}

\author{
N. Swapna ${ }^{1}$, B. Padmaja Rani ${ }^{2}$ \\ ${ }^{1}$ Research Scholar, Department of CSE, JNTU College of Engineering, Hyderabad, TS \\ ${ }^{2}$ Department of CSE, JNTU College of Engineering, Hyderabad, TS
}

\begin{abstract}
With the increasingly widespread use of computers and the internet in India, large amount of information in Indian languages are becoming available on the web. Automatic information processing and retrieval is therefore becoming an urgent need in the Indian context. This paper presents a new Rule based Pseudo N-gram for Telugu language. Rule based Pseudo N-gram is an approach, which provides a system that gives set of rules to extracting root words by removing inflections which were unrecognized by Pseudo N-gram. Pseudo N-gram can act as a preliminary stage for Rule based Pseudo N-gram. Pseudo N-gram is process of stripping the word from the end. We composed five rules to describe a Rule based Pseudo N-gram. The rules are written based on the morphology, grammar rules and word derivation structure of Telugu language. Telugu is one of the old and traditional languages of India and it is categorized as one of the Dravidian language family unit with its own high-class script. Telugu is an authorized language of the states of Telangana and Andhra Pradesh. Telugu is a rich morphological large that has high word conflation. Keeping in view of these complexities, we propose a Rule based Pseudo N-gram that provides a reasonable alternative to word based models and is also used for text categorization. We have conducted the experiments on randomly selected Telugu documents and we found the accuracy of Rule based Pseudo N-gram is up to 97.8\%.
\end{abstract}

Key words: Rule Based Pseudo N-Gram, Pseudo N-Gram, Text Categorization, Morphology, Grammar Rule. $* * *$

\section{INTRODUCTION}

Information Retrieval in local language is getting more popularity in developing countries like India. Automatic information processing and retrieval is therefore becoming an urgent need in the Indian context. Moreover, since India is a multilingual country, Telugu is the third most spoken languages in the world. It is the authorized language of the states of Telangana and Andhra Pradesh. There is an also a vast increase in Telugu language text documents with use of computers and the internet. When users need to retrieve some information from the Telugu language document depending on the query, they may get some irrelevant information (or) may lose some important information. Processing of Telugu documents is more difficult, because of the complexity when compared to European and other Indian languages. Telugu is morphologically rich language which contains too many suffixes and inflections. Building efficient information retrieval system for Telugu is a challenging task due to the richness in morphology and high word conflation feature of the language. The canonical structure is described as ( ( C ) C ) C V. The Telugu structure evolves with a set of syllables known as vowels and consonants, where consonant vowel ( CV) core is the basic unit optionally preceded by one (or) two consonants. They alone form independent syllables. Fig.1 (a) shows that Telugu language has 13 vowels and 36 consonants, Fig.1 (b) shows Telugu gunintamulu. In this paper, we proposed a Rule based Pseudo N-gram for Telugu language to extract the root word, which were unrecognized by pseudo N-gram. In this approach, Pseudo $\mathrm{N}$-gram can act as preliminary stage. Pseudo N-gram is the process of finding the root word by stripping the word from the end. Stripping length will be varied based on word length. Maximum stripping length is 5 and minimum is 2.Pseudo $\mathrm{N}$-gram model is an alternative to $\mathrm{N}$-gram and word based language models. We composed five rules to describe a Rule based Pseudo N-gram. These rules are used to replace the characters (or) syllables, when the words are not recognized by Pseudo N-gram.

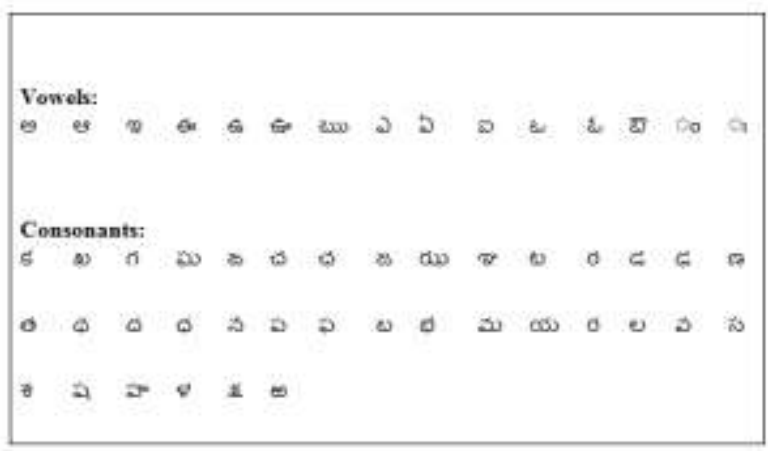

Fig 1 (a) Telugu vowels and consonants

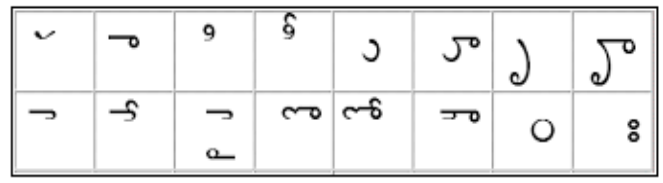

Fig 1 (b) Telugu Gunintamulu

\section{RELATED WORK}

Telugu is one of the old and traditional languages of India. Singhal Amit et al [4] surveyed that, in India the Telugu native speakers are above 50 millions. Many researchers and linguists built Telugu IR system. Pingali V.V Prasad et al [6] stated that, the Telugu IR work is at the initial level. Because of lagging of the resources for Telugu causes poor 
growth in information retrieval and in it's applications[5]. Telugu is a rich morphological language that has high word confloation where the word logic disambiguation can't be, resolved easily[7]. Padmaja Rani et al [9] proposed the search engine for Telugu document retrieval, which is experimented using syllable $\mathrm{N}$-gram model. In this approach a good transliteration system had been adapted and they have showed that the N.-gram model with length-3 will enhance the search capacity. Vishnu Vardhan et al [2] proposed the overlapping trigram technique for Telugu text categorization. A Durga $\mathrm{k}$ et al [8] projected a technique with ontology text categorization for Telugu digital-items and retrieval system. Sunitha, kalyani et al [3] suggested a approach to improve the performance of Telugu rule based Morphological analyzer. Pseudo N-gram is new method that reduces words with the same root to a common form, usually by removing derivational and inflectional suffixes from each word [1].It is an alternative to stemming, Ngraming and other word based models. For the best of our knowledge , Pseudo N-gram is the introductory approach has been hired on extracting Telugu root words.

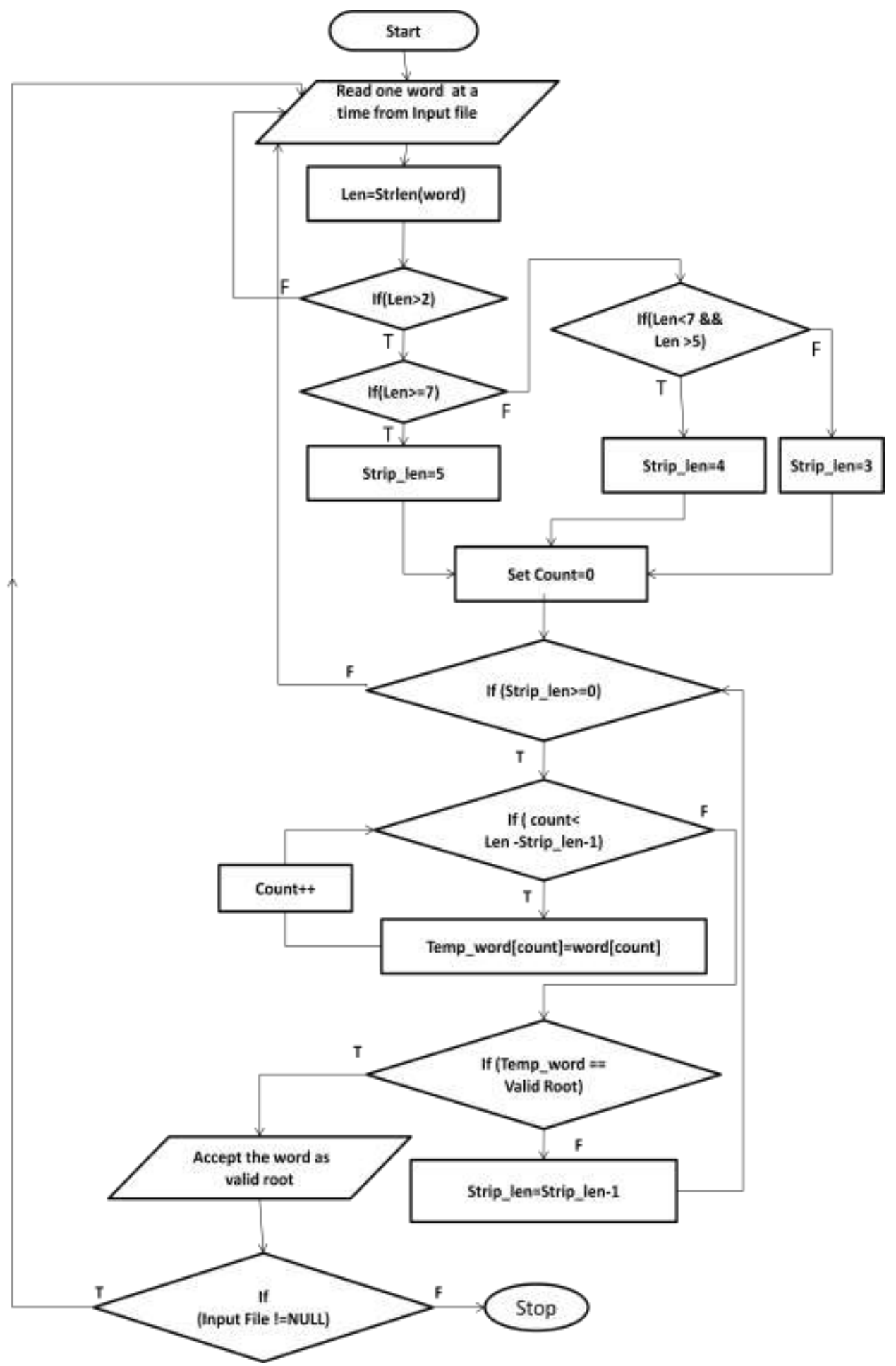

Fig.2 Flowchart of Pseudo N-gram 


\section{PSEUDO N-GRAM}

Pseudo $\mathrm{N}$-gram is a procedure that reduces words by stripping derivational and inflectional suffixes from each word to get valid root[1]. It takes raw words from Input File as input. Read one word at a time from file. First find the length of word and fix the stripping length. Stripping length will be varied based on word length. Maximum stripping length is 5 and minimum is 2 , and then apply Pseudo Ngram algorithm for each word. For each step, strip the word from end and check, if it is valid root or not. If it is valid root, then extract root, accept and perform categorization is shown in fig. 3 If it is not a valid root, decrease the stripping length by one and check. This process is repeated until stripping length not equals to zero. Fig.2 shows the flowchart of Pseudo N-gram.

\section{PROPOSED SYSTEM}

Pseudo N-gram is a base method for this processing to remove suffixes from words. The result of Pseudo N-gram of some words normally contains inflections. The inflections in the stem word cannot be removed using simple Pseudo Ngram. We have designed rule based Pseudo N-gram algorithm, which is shown in Fig .3 for some possible suffixes that frequently occur in the Telugu Language. The rules are used to replace characters. These rules are shown in Table 1.

\subsection{Rules for Rule based Pseudo N-gram}

Table 1. Rules for Rule based Pseudo N-gram

\begin{tabular}{|c|c|c|c|c|}
\hline S.No & $\begin{array}{l}\text { List of characters/syllable } \\
\text { sound found as suffix }\end{array}$ & $\begin{array}{l}\text { Replacement } \\
\text { characters }\end{array}$ & $\begin{array}{c}\text { After } \\
\text { Pseudo N- } \\
\text { gram }\end{array}$ & $\begin{array}{l}\text { After Rule based } \\
\text { Pseudo N-gram }\end{array}$ \\
\hline 1 & e,es & $\Theta \circ, \epsilon, Q$ & 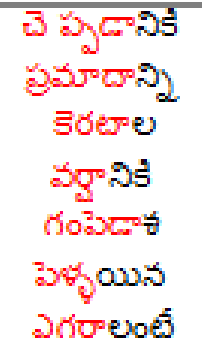 & $\begin{array}{l}\text { చెప్పడం } \\
\text { ప్రుూదం } \\
\text { కరటం } \\
\text { వర్వం } \\
\text { గంపెడు } \\
\text { పెళ్ళి } \\
\text { ఎగురు }\end{array}$ \\
\hline 2 & $Q, \infty$ & $\epsilon$, అ, అం & $\begin{array}{c}\text { నికెదందచడం } \\
\text { దేవుడ } \\
\text { హాంసీంచే }\end{array}$ & $\begin{array}{l}\text { నేపీదం } \\
\text { దేపుడు } \\
\text { హాంసు }\end{array}$ \\
\hline 3 & $\epsilon, \epsilon+e, \delta+e \omega$ & Q, అ০ & 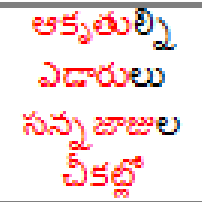 & 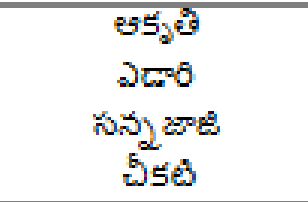 \\
\hline 4 & ৯,ఏ, ఎ০ & Q, & 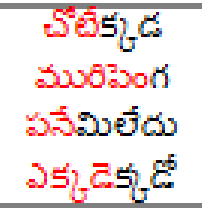 & $\begin{array}{c}\text { కాలు } \\
\text { మురం } \\
\text { పని } \\
\text { ఎక్కడ }\end{array}$ \\
\hline 5 & అం & $\epsilon$ & 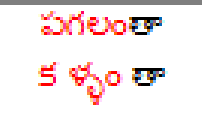 & $\begin{array}{l}\text { shes } \\
5 \% 0 y\end{array}$ \\
\hline
\end{tabular}




\subsection{Rule based Pseudo N-gram Algorithm}

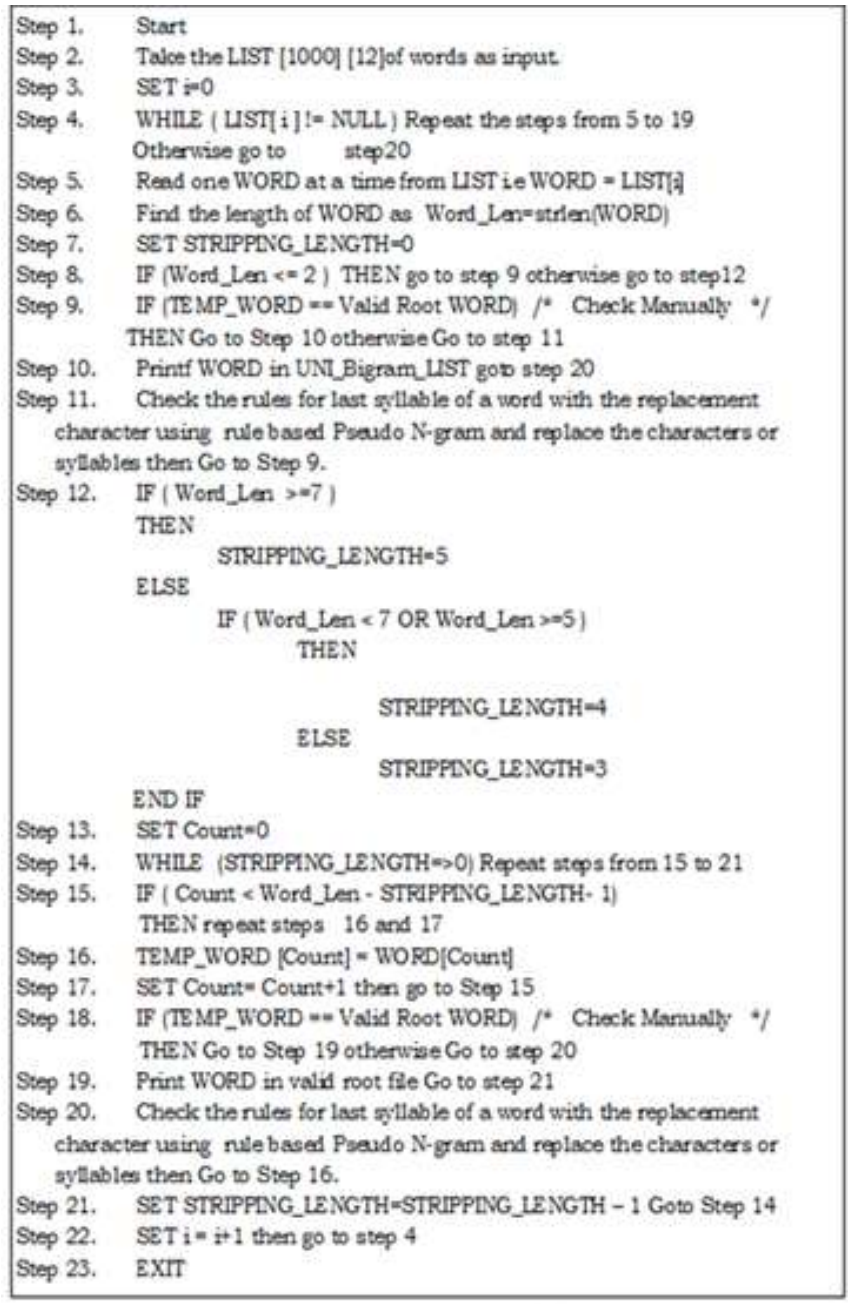

Fig.2 Algorithm of Rule based Pseudo N-gram
After Pseudo N-gram, if the word ends with " దిం, డి,

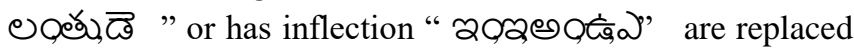
by the character " దQ డు,లు ఏి, డ " with inflection "అం $\epsilon_{\oplus}, \curvearrowright$, అ ". We use above rules to get the root for inflected word.

a) List of all the possible words which are not identified as root word using pseudo N-Gram.

b) Apply Pseudo N-gram and check the rules for inflected words to be replaced by another character.

c) The replacements characters to be made after removal of suffix character so that valid root can be formed.

\section{EXPERIMENTAL RESULTS}

The experiments were conducted on Telugu Corpus, collected from online newspapers and wikipedia.This work has been implemented on sample selection of 1,550 documents. A sequenece of words which are not recognized by pseudo $\mathrm{N}$-gram word list was used in extracting valid root by Rule based pseudo $\mathrm{N}$-gram algorithm and the results are presented in Table 2,which contains list of words after pseudo $\mathrm{N}$-gram and Rule based pseudo $\mathrm{N}$-gram with suitable rules for evaluation.

Table 2. Results of Rule based Pseudo N-gram algorithm

\begin{tabular}{|c|c|c|c|}
\hline $\begin{array}{l}\text { List of words before } \\
\text { pseudo N-gram }\end{array}$ & $\begin{array}{l}\text { List of words, which are } \\
\text { not recognized as valid root } \\
\text { after pseudo N-gram }\end{array}$ & $\begin{array}{l}\text { Rule for replacement } \\
\text { character }\end{array}$ & $\begin{array}{l}\text { Valid root words after } \\
\text { Rule based pseudo N-gram }\end{array}$ \\
\hline పర్వ తానికి & పర్వతా & Rule 1 & పర్వ తం \\
\hline ప్రయछ్నాలు & ప్రయత్వా & Rule 1 & ప్రయత్ప్రం \\
\hline దంతాలతో & దంతాల & Rule 1 & దంతాలు \\
\hline తీరాక & తీరా & Rule 1 & తీరు \\
\hline వాడిగ్ళృతో & వాడిగోళ్ & Rule 1 & వాడిగోళ్ళ \\
\hline శోకాలు & శ్రాకా & Rule 1 & శోకo \\
\hline కారేట్లుగా & కారే & Rule 4 & కారు \\
\hline విజృంభించి & విజ్ృభిం & Rule 2 & విజృంభం \\
\hline ఫలవృకాలతో & పలవృక్ & Rule 1 & పలవృక్ \\
\hline బయులుదేరింది & బయులుదేరిం & Rule 2 & బయులుదేరు \\
\hline జలాశ్రయాలకు & జలాశ్రయా & Rule 1 & జలాశ్రయం \\
\hline ఈరీరమంతా & ซరీర & Rule 1 & శరీరం \\
\hline ఘీంకారానికి & ఫీంకారా & Rule 1 & ఘీంకారం \\
\hline రక్కేరి & రక్కే & Rule 4 & రక్కు \\
\hline
\end{tabular}




\section{CONCLUSION}

Rule based pseudo N-gram technique is also well suited for different complex Indian languages like Hindi, Malayalam and Kannada. Our proposed approach will minimize inflections of words; it will become easy for retrieving desired information. In this paper, accuracy of Rule based pseudo $\mathrm{N}$-gram is more than pseudo $\mathrm{N}$-gram model. In my knowledge, there is no such report of Extracting Telugu language valid root words using pseudo N-gram and Rule based pseudo $\mathrm{N}$-gram. The maximum accuracy observed is 98\% for Rule based pseudo N-gram. As part of our research work in Telugu categorization, we propose to extend it for recognize all words as valid roots.

\section{REFERENCES}

[1] Porter M.F. “ An algorithm for suffix stripping”,pogram,14(3),1980,130-137

[2] B.vishnu varthan, L Pratap Reddy,A Vinay babu "A Model for overlapping trigram Technique for telugu script", Journal of Theoretical and applied information Technology, (JAJIT 2007).

[3] Kalyani, N. and Sunitha, K.V.N. (2009 ): A Novel approach to Improve rule based Telugu Morphological Analyzer, World Congress on Nature \& Biologically Inspired Computing (NaBIC 2009).

[4] Singhal, Amit, Modern Information Retrieval: A Brief Overview, Bulletin of the IEEE Computer Society Technical Committee on Data Engineering, Vol-24, issue-4, 2001, PP:35-43.

[5] N.Murthy, P Srikanth, Named entity recognition for Telugu, Proceedings of the IJCNLP-08 Workshop on NER for South and South East Asian Languages, 2008, PP:41-50.

[6] Pingili V.V Prasad, Recall Oriented Approaches for Improved Indian Language Information Access. Ph.D Thesis, 2009.

[7] G.U.Rao, 2008, Functional Specifications of Morphology CLATS, Hyderabad Central University, Version 1.3.1, 2008, PP:1-32.

[8] Mrs.A.Kanaka Durga, Dr.A.Govardhan, Ontology Based Text Categorization Telugu Documents, International Journal of Scientific \& Engineering Research Volume 2, Issue 9, September- 2011 , PP: 1-4

[9] Rani, Dr B. Padmaja, and Dr A. Vinay Babu. , Novel Implementation of Search Engine for Telugu Documents with Syllable N-Gram Model, International Journal of Engineering Science and Technology, vol- 2, issue-8, 2010, PP:3712-3720.

\section{BIOGRAPHIES}

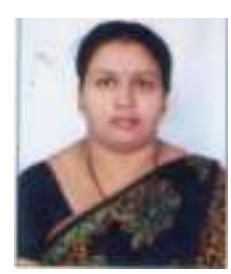

N. Swapna received B.Tech. in Computer Science and Information Technology from VREC-Nizamabad, JNT University. M.Tech. in Computer Science \& Engineering from JNTU Anantapur and she is Pursuing Ph.D. in the area of Information Retrieval Systems from the Department of Computer Science and Engineering, JNT University Hyderabad. She has 12 years of teaching experience in various engineering colleges. Currently she is working as Associate Professor and Training \& Placement Officer in the department of Computer Science Engineering ,Vijay Rural Engineering College, Nizamabad, India. To her credit Mrs.Swapna Narala has 10 ublications in various National / International Conference and Journals. She is also a Member of Various Technical Bodies including IEEE, ISTE etc. Her area of interest includes Information Retrieval, Text Mining, Web Mining, Machine Learning, Information Security etc.

B. Padmaja Rani received B.Tech Electronics Engineering

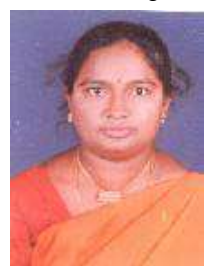
from Osmania University, M.Tech in Computer Science from JNT University Hyderabad, India and she has been awarded Ph.D. in Computer Science from JNT University, Hyderabad, India. At present she is working as Professor in the Department of Computer Science and Engineering, JNTUH College of Engineering, JNTU University Hyderabad. She is having 20 years of experience in Industry and Academia. At present she is a Professor of Computer Science and Engineering Department in JNTUH College of Engineering, JNT University, Hyderabad. Her area of Research includes Information Retrieval, Data Mining, Machine Translation, Computer Networks, Software Engineering etc. She is guiding 6 Research Scholars in the area of Information Retrieval and Computer Networks. To her credit she is having more than 60 publications in reputed International Journals and Conferences. She is a member of various advisory committees and Technical Bodies. She is also a Member of Various Technical Associations including ISTE, CSI, IEEE etc. 